\title{
Anisotropic Diffusion with Deep Learning
}

\author{
Hyun-Tae $\mathrm{CHOI}^{1}$, Yuna HAN, Dahye KIM, Seonghoon HAM, Minji KIM, \\ Yesol PARK, and Byung-Woo HONG \\ Computer Science Department, Chung-Ang University, Seoul, Korea
}

\begin{abstract}
We propose a deep learning framework for anisotropic diffusion which is based on a complex algorithm for a single image. Our network can be applied not only to a single image but also to multiple images. Also by blurring the image, the noise in the image is reduced. But the important features of objects remain. To apply anisotropic diffusion to deep learning, we use total variation for our loss function. Also, total variation is used in image denoising pre-process.[1] With this loss, our network makes successful anisotropic diffusion images. In these images, the whole parts are blurred, but edge and important features remain. The effectiveness of the anisotropic diffusion image is shown with the classification task.
\end{abstract}

Keywords. anisotropic diffusion, total variation, classification, deep learning

\section{Introduction}

Many approaches to object classification make essential use of machine learning methods. To improve the accuracy, we collect larger datasets, make more complicated models, and pre-process the training, testing dataset. But from the pre-processing point of view, the noise of image is a big problem to train and test a deep learning network model. There is a lot of research to reduce noise in images.[2] Like other denoising with deep learning, we try to decrease the noise in the image by diffusing using our deep learning network. In our experiments, we define noise as not important information for the classification task. For image denoising, we make a network that executes Anisotropic Diffusion. Filters in anisotropic diffusion can outperform isotropic diffusion to certain applications such as denoising of highly degraded edges.[3] And anisotropic diffusion reduces noise in the image without removing significant parts of the image content, typically edges[4]. With anisotropic diffusion, we can reduce the noise of the image and blur unnecessary parts of the image except the edge. It resembles the process that creates a scale space[3] where an image generates a parameterized family of successively more and more blurred images based on the diffusion process. This anisotropic diffusion is a generalization of this diffusion process. To observe the effect of our anisotropic diffusion image result, we check the classification accuracy.

Section 2 reviews anisotropic diffusion and its traditional algorithm. Section 3 describes the training process of our network. Section 4 includes the experiment results of our experiment. Finally, section 5 finish the paper by summarizing.

\footnotetext{
${ }^{1}$ Corresponding Author: E-mail: hyuntae@image.cau.ac.kr
} 


\section{Anisotropic diffusion}

Anisotropic diffusion reduces noise in the image without removing significant parts such as the edge. To make this image, anisotropic diffusion produces a family of parameterized images. Each resulting image is a combination of original images and a filter that depends on the local content of the original image. So anisotropic diffusion is a nonlinear and space-variant transformation of the original image[5]. To produce a family of parameterized images, anisotropic diffusion iterates the algorithm under the assumption that 'Inside of edge will be the same area'. This is the simple algorithm of anisotropic diffusion.

\section{Algorithm 1}

\section{for number of training iterations do}

1. Calculate edge gradient in four directions based on the current pixel.

2. In each direction, generalize it using generalization constant and sum it.

3. Output sum of the above-calculated values and weights(Lambda).

The traditional anisotropic diffusion algorithm works with Eq. (1).[4] In Eq. (1). $\nabla$ denotes gradient, $\Delta$ denotes the Laplacian, div is the divergence operator and $\mathrm{c}(\mathrm{x}, \mathrm{y}$, t) is the diffusion coefficient that controls the rate of diffusion. It is usually chosen as a function of the image gradient. Because of this coefficient, anisotropic diffusion can preserve edges in the image.

$$
\frac{\delta I}{\delta t}=\operatorname{div}(c(x, y, t) \nabla I)=\nabla C \cdot \nabla I+c(x, y, t) \Delta I
$$

But the traditional anisotropic diffusion algorithm is just for a single image and the speed is too slow. So, we apply anisotropic diffusion to deep learning framework for generalization and faster than the traditional algorithm.

\section{Training}

\subsection{Anisotropic Diffusion Loss}

To calculate the gradient of each direction, we try to calculate the total variation of anisotropic diffusion image which is the output of our network. Given an image $I$, the output of the anisotropic diffusion image is $U$. The loss function is defined as Eq. (2):

$$
\begin{aligned}
& \text { Loss }=\|U-I\|^{2}+\lambda\|\nabla U\| \\
& \|\nabla U\|=\left\|\nabla U_{x}\right\|+\left\|\nabla U_{y}\right\|
\end{aligned}
$$


$\lambda$ is the regularization parameter. $\nabla U_{x}$ is the gradient in the $\mathrm{x}$-axis direction of the anisotropic diffusion image and $\nabla U_{y}$ is the gradient in the y-axis direction of the anisotropic diffusion image. In this loss function, $\lambda$ term makes the output image is not too blurred. If this value is too big, the output image is too blurred to recognize the objects in the image. And if this value is too small, the output image is the same as the original image. So we set this value to 0.01 through several experiments. To calculate $\|\nabla U\|$ in Eq. (2), we calculate Eq. (3). To get the value of $\left\|\nabla U_{x}\right\|$ and $\left\|\nabla U_{y}\right\|$, we use CentralDifference with Dirichlet boundary condition. With this $\|\nabla U\|$, we can compute the total variation of network output. For this output images, our network is Auto-encoder network with 4-layers for encoding, and 4-layers.[6] We use the Auto-encoder structure for decoding to force the learned representations of the input to have useful properties.[7] In this architecture, the regularization parameter is fixed to 0.01 , the filter size is $3 \times 3$, and the input, output image size is $256 \times 256$.

\subsection{Classification}

To compare the accuracy of classification with the original image and anisotropic diffusion image, we train three networks with the same structure. The first network is trained with the original images and tested with the original images. The second network is trained with anisotropic diffusion images and tested with anisotropic diffusion images. And the third network is trained with CIFAR-10(Canadian Institute For Advanced Research) [8] and tested with anisotropic diffusion images of the CIFAR-10 test dataset. We use VGG-19(Visual Geometry Group)[9] network for classification network to compare the results.

\section{Experiment Result}

\subsection{Anisotropic Diffusion Network}

We train our network with the celeba dataset[10]. Celeba dataset is a collection of celebrity faces with a simple background and their faces are clear to recognize. So it is not too complicated. And face has distinct features such as eyes, nose, and mouth. So with this celeba dataset, we can compare the original image and anisotropic diffusion image to see whether important features remain after the denoising process. Figure 1 illustrates the original celeba image and its anisotropic diffusion celeba image from our anisotropic diffusion network. With anisotropic diffusion, the whole image is blurred except the edge of objects in the image. Although the image is blurred, we can still observe the eyes, nose, and mouth. So, noise in the image can be reduced with anisotropic diffusion by blurring. Also, distinct features remain, so there is no problem in recognizing the object. Additionally, to see whether the network works on more complex images, we use the BSDS dataset(Berkeley Segmentation Dataset) which is usually used for segmentation and the PASCALVOC dataset(PASCAL Visual Object Classes) which is usually used for object detection.[11][12] They are more complicated than celeba dataset. Figure 2 illustrates the result of the BSDS dataset and Figure 3 illustrates the result of the PASCALVOC dataset. With this Figure 2 and Figure3, we can see that our anisotropic diffusion network also works on a more complex image dataset. In each pair of images, 
the left side is original images and the right side is anisotropic diffusion images. The anisotropic diffusion image is blurred than the original images. As we can see with BSDS and PASCALVOC anisotropic diffusion images results, the whole images are blurred but the shape of objects in images remains. Despite the data becoming more complex, the anisotropic diffusion result images still have their object's shape. Therefore, we could see that even complex images could be applied to our network.
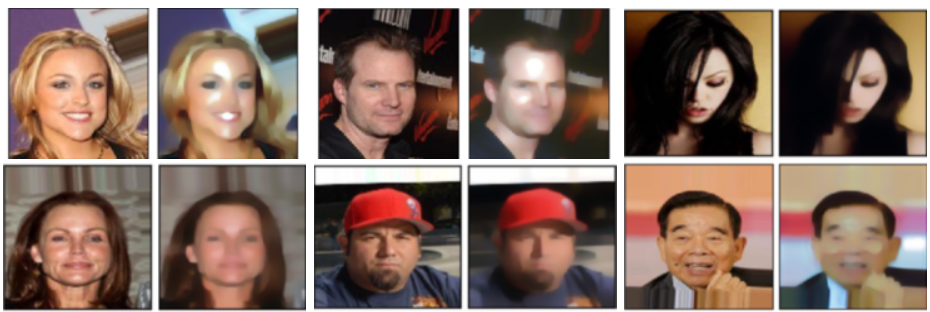

Figure 1. In each pair of images, the left side is the original celeba image and the right side is the anisotropic diffusion celeba image.
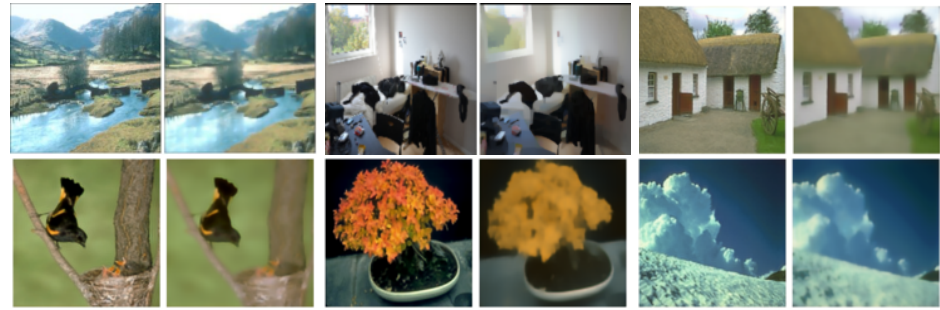

Figure 2. In each pair of images, the left side is the original BSDS image and the right side is the anisotropic diffusion BSDS image.
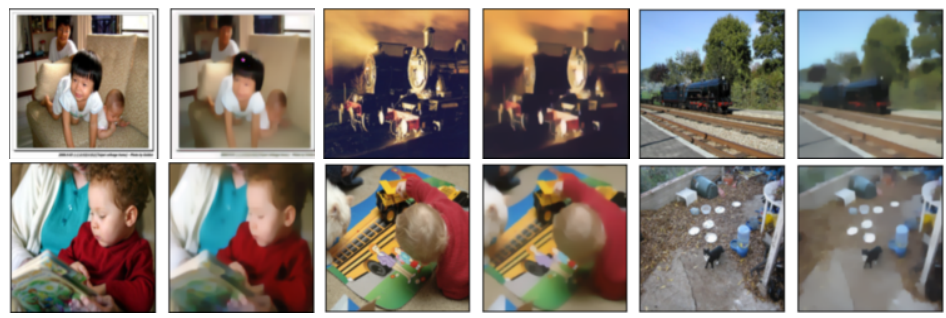

Figure 3. In each pair of images, the left side is the original PASCALVOC image and the right side is the anisotropic diffusion PASCALVOC image. 


\subsection{Classification}

To see the anisotropic diffusion image is effective, we use the result of anisotropic diffusion for three experiments with the CIFAR-10 dataset and VGG-19 network. Table 1 illustrates the classification accuracy $(\%)$ results. With case 1 and case 2 , we can see that the anisotropic diffusion image of the original is slightly more effective for classification. Also with case 1 and case 3 , even the network is trained with the original image, test accuracy with anisotropic diffusion image is higher than the original image. With this result and Figure 1, anisotropic diffusion reduces noise in the image by blurring the original image but remains important features. So important information about the objects in the image is preserved by this anisotropic diffusion method.

Table 1. Train and Test accuracy(\%) with CIFAR-10 and anisotropic diffusion image of CIFAR-10. Case 1 train and test with CIFAR-10. Case 2 train and test with anisotropic diffusion image. Case 3 train with CIFAR10 and test with anisotropic diffusion image of the CIFAR-10 test set.

\begin{tabular}{ccc}
\hline & Train Accuracy & Test Accuracy \\
\hline Case 1 & 88.16 & 77.56 \\
Case 2 & 90.24 & 83.27 \\
Case 3 & 88.16 & 80.94 \\
\hline
\end{tabular}

\section{Conclusion}

The traditional algorithm iterates its process with a single image.[13] So the diffusion coefficient and other parameters are suitable for only one image. And the speed of the algorithm is too slow. But with this our deep learning anisotropic diffusion network, we can apply it to other datasets not just for a single image. Also, by blurring the image, unnecessary information is reduced but the important features of the object remain. We show the effect of our result with classification accuracy using anisotropic diffusion image, that the difference from the accuracy of the original image is not so big and even better. With this result, the anisotropic diffusion image can be used to classification tasks instead of the original image. Even, although we use the VGG-19 network that is a very simple network for classification, test accuracy is slightly higher than the original image. It proves that anisotropic diffusion images still have important features and can be used for the classification task. Our result is simple, but it shows that anisotropic diffusion image can be used in other areas such as object tracking. Because object tracking does not need whole information of the image. It just needs important features that can represent the object. Our experiments show that anisotropic diffusion with deep learning reduces noise in the images but still have important features of objects.

\section{Acknowledgement}

This research was supported by the MSIT(Ministry of Science and ICT), Korea, under the National Program for Excellence in SW)(20170001000041001) supervised by the IITP(Institute of Information \& communications Technology Planning \&Evaluation) 


\section{References}

[1] Rudin LI, Osher S, Fatemi E. Nonlinear total variation-based noise removal algorithms. 1992.

[2] Tian CW et al. Deep learning on image denoising: An overview. arXiv preprint arXiv:1912.13171, 2019.

[3] Weickert J Anisotropic diffusion in image processing. Stuttgart: B.G. Teubner; 1998:5-8.

[4] Perona P and Malik J. Scale-space and edge detection using anisotropic diffusion. 1990. pp. 629-639

[5] Joachim W. A review of nonlinear diffusion filtering. Scale-Space Theory in Computer Vision. Springer, LNCS 1252; 1997. pp. 1-28.

[6] Baldi P. Autoencoders, unsupervised learning, and deep architectures. In: Proceedings of ICML workshop on unsupervised and transfer learning; 2012. pp.37-49.

[7] Goodfellow I, Bengio Y, Courville A. Deep Learning. MIT Press; 2016. 499 p.

[8] Krizhevsky A, Hinton G. Learning multiple layers of features from tiny images. Technical report, University of Toronto; 2009. 3 p.

[9] Simonyan K, Zisserman A. Very deep convolutional networks for large-scale image recognition.2014.

[10] Liu ZW et al. Large-scale celebfaces attributes (celeba) dataset. Retrieved August, 2018, 15: 2018.

[11] Arbelaez P, Fowlkes C, Martin D. The Berkeley segmentation dataset and benchmark. Computer Science Department, Berkeley University. 2007.

[12] Everingham M, Eslami SMA, Van Gool L, Williams CKI, Winn J and Zisserman A. Int J Comp Vision, 2015, 111(1), 98-136.

[13] Yu YJ, Acton ST. Speckle reducing anisotropic diffusion. IEEE Transactions on image processing, 2002, 11.11: 1260-1270. 